\title{
Characteristics and Long-Term Outcome of Neurosarcoidosis: A Population-Based Study from 1976-2013
}

\author{
Patompong Ungprasert ${ }^{a, b}$ Cynthia S. Crowson ${ }^{b, c}$ Eric L. Matteson ${ }^{b, d}$ \\ ${ }^{a}$ Division of Rheumatology, Department of Medicine, Faculty of Medicine Siriraj Hospital, Mahidol University, \\ Bangkok, Thailand; ${ }^{\mathrm{b}}$ Division of Rheumatology, Department of Internal Medicine, ${ }^{\mathrm{C}}$ Division of Biomedical Statistics \\ and Informatics, and d Division of Epidemiology, Department of Health Science Research, Mayo Clinic College of \\ Medicine and Science, Rochester, MN, USA
}

\section{Keywords}

Sarcoidosis · Neurosarcoidosis · Epidemiology · Outcome

\begin{abstract}
Background/Aims: Neurosarcoidosis is a rare condition with serious health consequences. However, little is known about clinical characteristics and outcome of neurosarcoidosis in the community setting. Methods: Patients with neurosarcoidosis were identified from a previously described cohort of patients with incident sarcoidosis from Olmsted County, Minnesota, United States from 1976 to 2013 using individual medical record review. Data on clinical characteristics, treatment, and outcome were collected. Results: Neurological involvement by sarcoidosis occurred in 11 patients ( $3 \%$ of all patients with sarcoidosis). Cranial neuropathy was the most common type of neurological disease (5 patients; $45 \%$ ) followed by peripheral neuropathy ( 3 patients; $27 \%$ ), and meningitis (3 patients; $27 \%$ ). Cerebrospinal fluid (CSF) pleocytosis and elevated CSF protein levels were observed in patients with meningitis, intramedullary spinal cord sarcoidosis, intracranial mass lesion and some patients with cranial neuropathy but were normal in patients with peripheral neuropathy. All patients received high-dose glucocorticoids as initial treatment and almost all responded to this therapy.
\end{abstract}

Relapse after glucocorticoid dose reduction necessitated subsequent treatment with steroid-sparing agents in 4 patients. Conclusion: Neurosarcoidosis is an uncommon manifestation of sarcoidosis. Neurosarcoidosis manifestations generally responded well to high-dose glucocorticoids in the majority of patients, but relapse was common.

C 2017 S. Karger AG, Base

\section{Introduction}

Sarcoidosis is an immune-mediated disorder characterized by the presence of noncaseating granuloma. The exact pathogenesis of sarcoidosis is not known but is believed to be a dysregulated immunologic response to environmental trigger(s) $[1,2]$. The reported incidence of sarcoidosis ranges from 1 to 70 new cases per 100,000 person-years, depending on the ethnic background of the population [3-5]. Sarcoidosis can affect any organ, with the lung and mediastinal lymph node being the most frequently affected sites. Involvement of the nervous system by sarcoidosis is relatively uncommon with a reported prevalence of $4-9 \%[3,6-8]$ but is associated with high morbidity and mortality. The current study used a previously identified cohort of patients with sarcoidosis from

\section{KARGER}

(C) 2017 S. Karger AG, Basel

E-Mail karger@karger.com

www.karger.com/ned
Patompong Ungprasert, MD, MS

Division of Rheumatology

Mayo Clinic, 200 First avenue SW

Rochester, MN 55905 (USA)

E-Mail P.Ungprasert@gmail.com 
Olmsted County, MN, United States, to characterize the epidemiology, clinical characteristics, and outcome of patients with neurosarcoidosis [9].

\section{Methods}

This is a retrospective cohort study that identified potential cases of incident sarcoidosis from 1976 to 2013 from the medical record-linkage system of the Rochester Epidemiology Project (REP) using the diagnosis codes related to sarcoid, sarcoidosis, and noncaseating granuloma. The REP medical record-linkage system collects diagnostic codes of all clinical encounters (hospitalization, outpatient visit, and emergency room visit) of Olmsted County, Minnesota residents with local providers (the Mayo Clinic, the Olmsted Medical Center and their affiliated hospitals, local nursing homes and few private practitioners). Thus, virtually all clinically recognized cases of sarcoidosis in the community can be identified from the database. History, methodology, and clinical application of the REP medical record-linkage system have been described in detail elsewhere [10].

Medical records of the identified cases were individually reviewed to confirm the diagnosis of pulmonary sarcoidosis, which required the presence of noncaseating granuloma on histopathology, radiologic features of intrathoracic sarcoidosis, compatible clinical presentation, and exclusion of other possible causes of granulomatous inflammation such as tuberculosis and fungal infection. The only exception to the histopathology requirement was stage I pulmonary sarcoidosis that required only the presence of symmetric bilateral hilar adenopathy on thoracic imaging studies after excluding other alternative diagnoses. Isolated extrathoracic sarcoidosis (including isolated neurosarcoidosis) without pulmonary sarcoidosis was diagnosed by the presence of noncaseating granuloma, compatible clinical presentation, and exclusion of other granulomatous diseases, similar to the diagnosis of pulmonary sarcoidosis. However, diagnosis of all cases of extrathoracic sarcoidosis required histopathologic confirmation. Patients who were diagnosed with sarcoidosis prior to residency in Olmsted County (i.e., prevalent cases) were not included.

Confirmed cases of sarcoidosis were then reviewed for assessing neurologic involvement. Data on demographic characteristics, neurological manifestations, imaging study of the brain/spinal cord, cerebrospinal fluid (CSF) analysis, laboratory investigations, treatment, and outcome were collected. Follow-up was continued until death, migration out of system, or April 1, 2017. Descriptive statistics were used to summarize the data. This study was approved by the Mayo Clinic and the Olmsted Medical Center Institutional Review Boards and the need for inform consent was waived.

\section{Result}

Between 1976 and 2013, 345 residents of Olmsted County, MN were diagnosed with sarcoidosis. Demographics and clinical characteristics of this cohort were previously described in detail [9]. Neurological involve- ment was observed in 11 patients ( $3 \%$ of all patients with sarcoidosis; 95\% CI 2-6\%). Among these 11 patients, 55\% were female, $73 \%$ Caucasian, $18 \%$ African-American, and $9 \%$ Asian with an average age of 44.8 years (SD 10.7 years at diagnosis; Table 1). Most patients fulfilled the criteria for probable neurosarcoidosis as proposed by Zajicek et al. [11]. A total of 3 patients fulfilled the criteria for definite neurosarcoidosis and 1 patient fulfilled the criteria for possible neurosarcoidosis. Cranial neuropathy was the most common type of neurological disease (5 patients; $45 \%$ ) followed by peripheral neuropathy (3 patients; $27 \%$ ) and meningitis (3 patients; 27\%). Intramedullary spinal cord sarcoidosis and intracranial mass lesion were present in 1 patient. Extraneurologic involvement by sarcoidosis was also seen in all but 1 patient. Lung was by far the most commonly affected extraneurologic site (73\%) followed by skin (18\%). Involvement of skin, joint, lacrimal gland, and extrathoracic lymph node was seen in 1 patient.

Neurological abnormalities were part of the presenting symptoms that led to the diagnosis of sarcoidosis in 7 of the 11 patients (64\%). The median time from onset of neurological symptoms to diagnosis of sarcoidosis was 1 month (range 2 weeks to 4 months). Neurosarcoidosis occurred after the diagnosis of sarcoidosis was established in the other 4 patients. In 1 patient, neurosarcoidosis developed 22 years after the initial diagnosis of systemic sarcoidosis.

CSF analysis was performed in all but 1 patient. All of those with meningitis had CSF pleocytosis (cell count ranged from 37 to $107 \mathrm{cell} / \mathrm{mm}^{3}$, predominately lymphocytes; normal range $0-5$ cells $/ \mathrm{mm}^{3}$ ) and elevated CSF protein level (range 47-206 mg/dL; normal range 14-45 $\mathrm{mg} / \mathrm{dL}$ ), but hypoglycorrhachia was seen only in 1 patient. CSF pleocytosis and high CSF protein level were also observed in the patient with intramedullary spinal cord sarcoidosis, the patient with intracranial mass lesion, and in some patients with cranial neuropathy but not in patients with peripheral neuropathy. Oligoclonal bands were present in the CSF of only 1 patient (14\% of testes patients). The agiotensin-converting enzyme level was obtained in 10 patients and was elevated in 2 patients (20\%).

All patients, except for 1 with isolated cranial nerve VII neuropathy, were treated with prolonged courses of glucocorticoids of 1 year or more. Almost all patients had a good response to initial treatment with high-dose glucocorticoids, often initially $1 \mathrm{~g}$ methylprednisolone daily for 3-5 days, or prednisone, $1 \mathrm{mg} / \mathrm{kg}$ body weight as initial dose. However, relapse of neurosarcoidosis was 


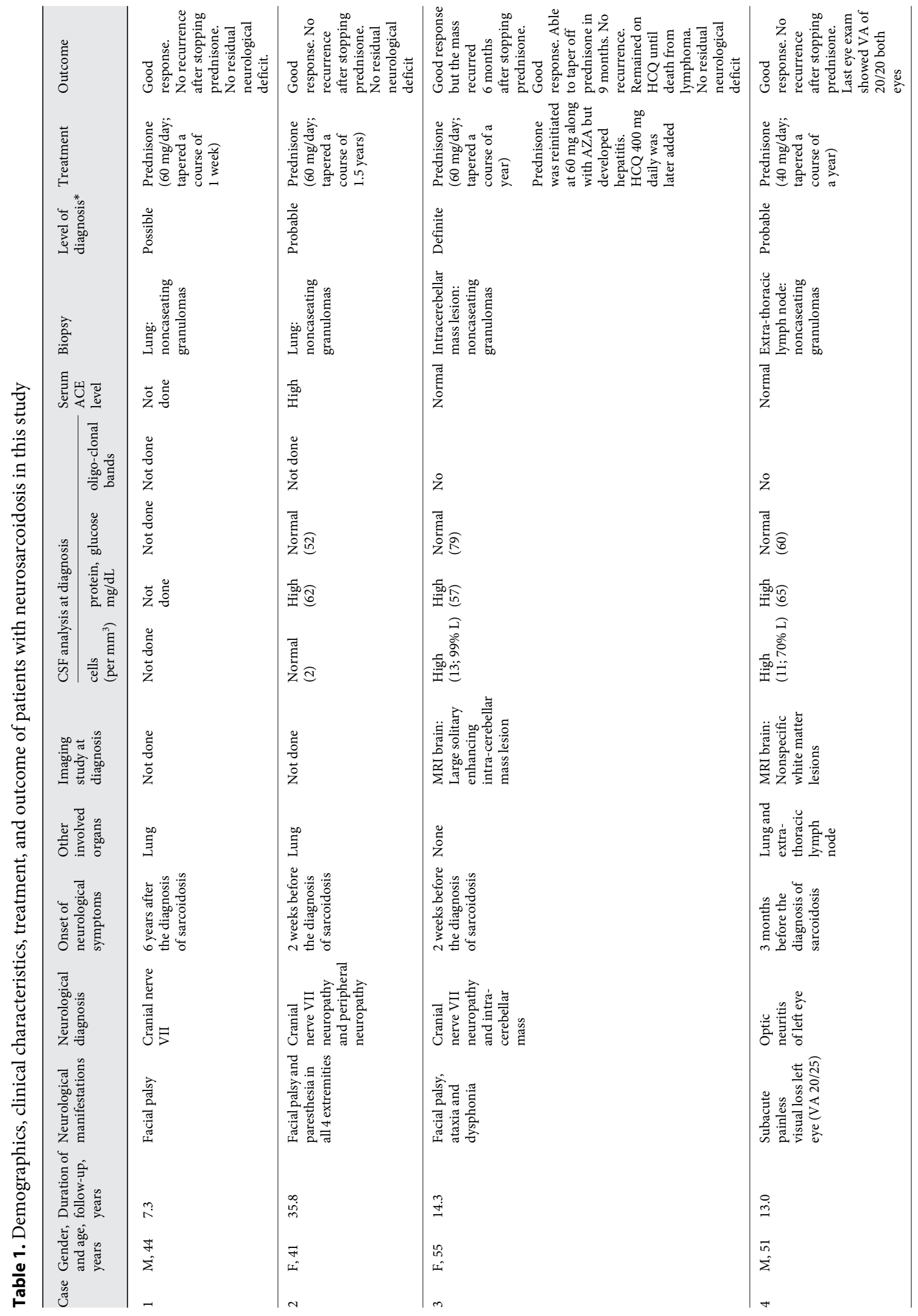




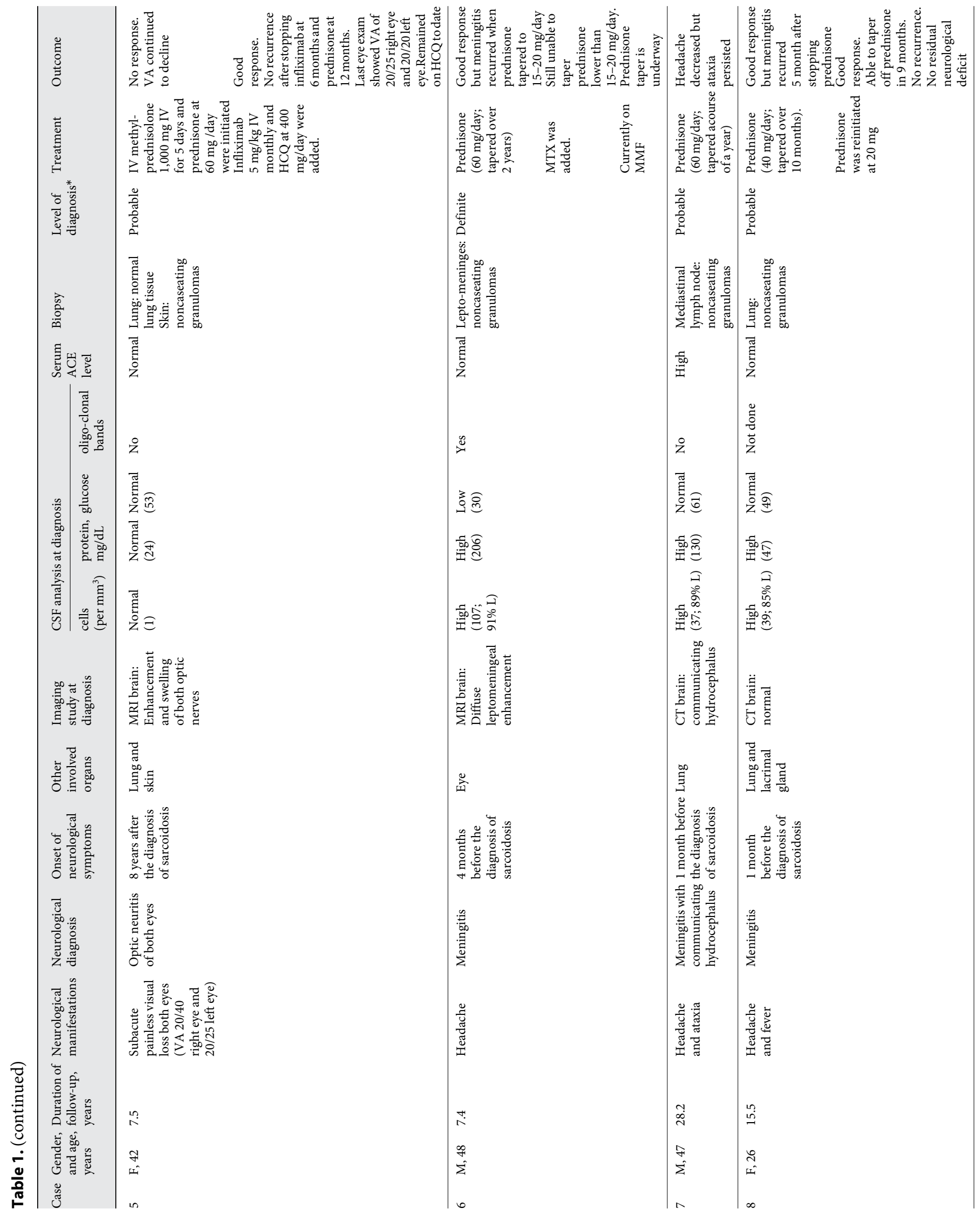




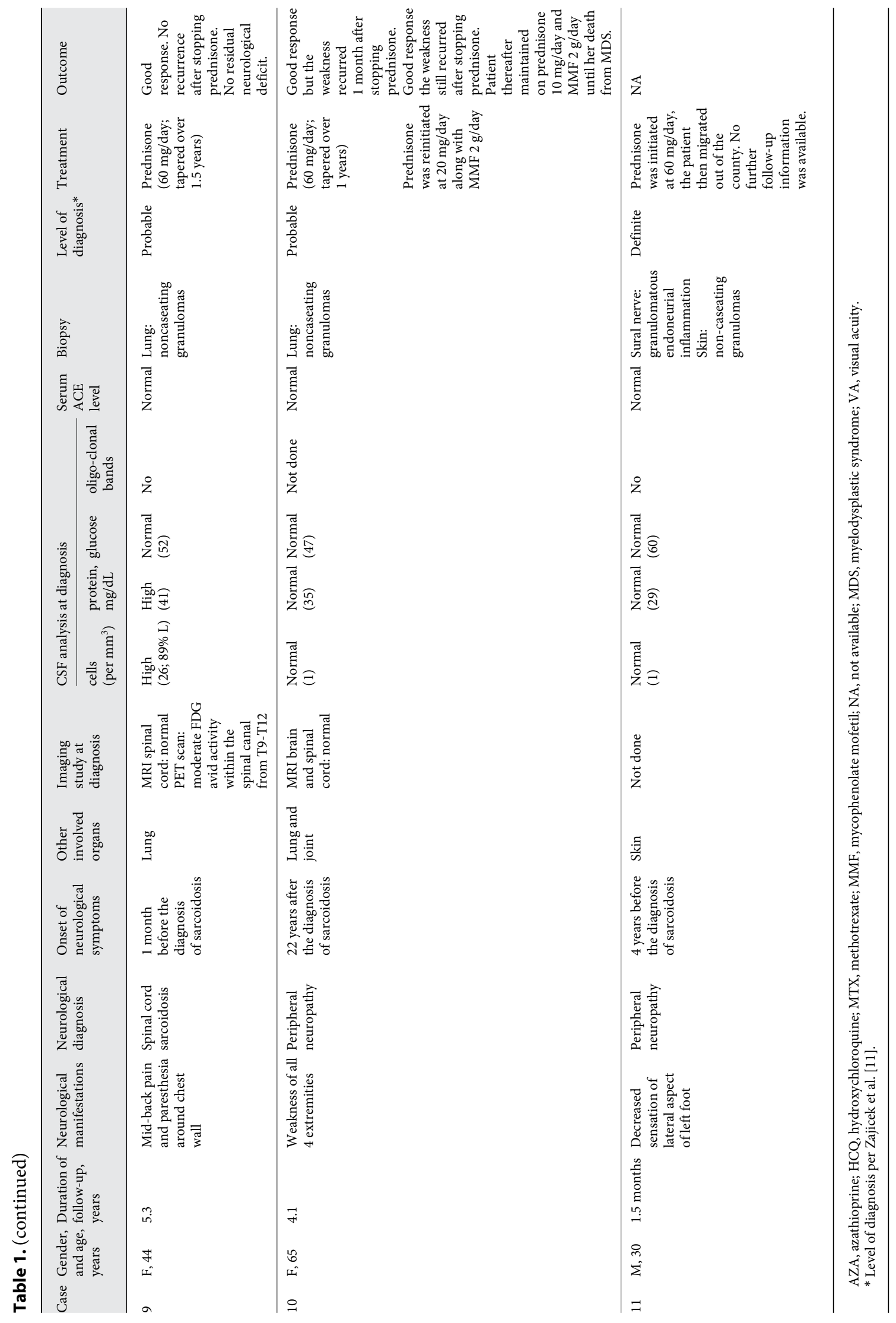


common (4 patients) after the treatment was discontinued or the glucocorticoid dose was reduced. Several disease-modifying anti-rheumatic disease drugs (DMARDs), including methotrexate, mycophenolate mofetil, hydroxychloroquine, and azathioprine were used in this cohort as glucocorticoid-sparing agents after failure of glucocorticoids taper. The disease progressed in 1 patient despite high-dose glucocorticoids. Infliximab was added as rescue therapy with good response.

\section{Discussion}

Similar to previous studies, the prevalence of neurosarcoidosis was low in the current cohort (3\% of all patients with sarcoidosis). However, the prevalence observed in this study is slightly lower than the reported prevalence of $4-9 \%$ from previous referral-based cohorts $[3,6-8]$. The difference in methodology is possibly responsible for the lower prevalence, as the current study utilized a population-based cohort of patients with sarcoidosis, which may be able to capture a more complete spectrum of sarcoidosis in the community, unlike referral-based studies that may have selection bias and include more severe cases. The frequency of specific types of neurological abnormalities in this cohort was similar to previous studies with cranial neuropathy being the most common neurological abnormality, present in almost half of patients, followed by meningitis and peripheral neuropathy $[12,13]$.

The diagnosis of neurosarcoidosis is often challenging as the neurologic abnormalities due to neurosarcoidosis can be seen in a myriad of neurologic disorders. Demonstration of noncaseating granuloma in neural tissue remains the gold standard for the diagnosis. However, biopsy of the nervous system is often prohibitively invasive [13]. In addition, false negative results are common, with up to $30-40 \%$ of brain parenchymal and meningeal biopsies failing to demonstrate the presence of noncaseating granuloma [14]. Zajicek et al. [11] proposed a set of criteria for diagnosing neurosarcoidosis that allows a "probable" diagnosis to be made by biopsy of an extraneurologic organ and evidence of inflammation in the central nervous system (from CSF analysis and/or MRI) without the requirement of nervous system histopathology. None of the patients in this cohort had isolated neurosarcoidosis. Sarcoidosis affected at least 1 additional organ in all patients in this cohort, with the lung being the most commonly affected organ.
The high frequency of extraneurologic sarcoidosis among patients with neurosarcoidosis has been consistently demonstrated in several cohorts $[11,13,15]$. Thus, careful physical examination and imaging study of the thorax should be performed to identify affected organs that may be eligible as alternative sites for biopsy. Histopathological confirmation of the diagnosis was available in all patients, including the biopsy of nervous system tissues in 3 patients. Identification of the extent of sarcoidosis is also important for monitoring the progression and response to treatment of the disease in each organ.

CSF analysis can be helpful to identify the central nervous system inflammation, as most patients with neurosarcoidosis involving the central nervous system in this cohort had CSF pleocytosis and elevated CSF protein level. However, the degree of CSF abnormality was generally mild (highest protein level in this cohort was $206 \mathrm{mg} / \mathrm{dL}$; highest cell count was $107 \mathrm{cell} / \mathrm{mm}^{3}$ ). In general, more profound CSF abnormalities including neutrophil-predominant pleocytosis and hypoglycorrhachia are not characteristics of neurosarcoidosis and are more suggestive of bacterial infection. Oligoclonal bands were detected in CSF only in 1 patient in this cohort. This observation is in line with the low prevalence of oligoclonal bands in other neurosarcoidosis cohorts $[11,16]$. CSF analysis for oligoclonal bands may have a diagnostic value for differentiating neurosarcoidosis from multiple sclerosis with their presence being suggestive of multiple sclerosis; their absence, on the other hand, is suggestive of neurosarcoidosis.

A high rate of relapse after initial response to high-dose glucocorticoids was observed in this cohort despite prolonged duration (1-2 years) of glucocorticoids treatment in almost all patients. A high recurrence rate was described in previous studies as well $[16,17]$. Several DMARDs were used as steroid-sparing agents in this study, but only 2 patients were able to discontinue glucocorticoids and both received hydroxychloroquine. Another study reported the stabilization and/or improvement of neurological symptoms in 10 out of 12 patients who could not tolerate or who were elected not to take glucocorticoids and were treated with hydroxychloroquine mono-therapy [18]. Given the significant morbidity associated with relapse, the use of concomitant DMARD therapy could be considered from the outset of treatment along with glucocorticoids, similar to the treatment of other autoimmune diseases such as rheumatoid arthritis and systemic lupus erythematosus $[19,20]$. The potential benefits of DMARD therapy must be weighed against their potential risks. Controlled studies are required to establish the role of combination therapy in neurosarcoidosis. 
The efficacy of infliximab, a chimeric monoclonal antibody against tumor-necrosis factor alpha, for pulmonary sarcoidosis has been demonstrated in randomized, controlled clinical trials $[21,22]$. In this cohort, infliximab was successfully used in 1 patient as a rescue therapy after glucocorticoids failure. The efficacy of infliximab as a rescue therapy for neurosarcoidosis was previously described in a few case reports and case series [23, 24], but no controlled study is available.

This study has some limitations related to its retrospective nature, as patients were not systematically evaluated for neurologic involvement of sarcoidosis. Therefore, the burden of unrecognized disease is not known. In addition, some recognized cases may not have been captured in this cohort if they were not correctly coded as sarcoidosis in the medical-linkage system. There was no standard follow-up or documentation protocol and, thus, some of the pertinent data were not available. Generalizability of the observations is another limitation, as the clinical characteristics and outcome of sarcoidosis vary considerably between ethnic groups [5]. The population of Olmsted County, MN as well as the patients in this cohort, is predominately of northern European ancestry.

\section{Conclusion}

Neurosarcoidosis is an uncommon manifestation of sarcoidosis that was seen in only $3 \%$ of patients in this cohort. Cranial neuropathy, meningitis, and peripheral neuropathy were the most common types of neurological diseases. Neurosarcoidosis manifestations generally responded well to high-dose glucocorticoids in the majority of patients, but relapse was common.

\section{Disclosure Statement}

The authors have no financial or nonfinancial potential conflicts of interest to declare.

\section{Funding Sources}

This study was made possible using the resources of the REP, which is supported by the National Institute on Aging of the National Institutes of Health (NIH) under Award Number R01AG034676, and CTSA Grant Number UL1 TR000135 from the National Center for Advancing Translational Sciences, a component of the NIH. The content is solely the responsibility of the authors and does not necessarily represent the official views of the NIH.

\section{Author Contribution}

P.U. was responsible for conception and design, acquisition, and interpretation of data, drafting of the manuscript, statistical analysis. E.L.M. was responsible for conception and design, acquisition, and interpretation of data, critical revision of the manuscript for important intellectual content, statistical analysis, and supervision. C.S.C. was responsible for conception and design, analysis and interpretation of data, critical revision of the manuscript for important intellectual content, and statistical analysis.

\section{References}

1 Hu Y, Yibrehu B, Zabini D, Kuebler WM: Animal models of sarcoidosis. Cell Tissue Res 2017;367:651-661.

2 Sanchez M, Haimovic A, Prystowsky S: Sarcoidosis. Dermatol Clin 2015;33:389-416.

3 Morimoto T, Azuma A, Abe S, Usuki J, Kudoh S, Sugisaki K, Oritsu M, Nukiwa T: Epidemiology of sarcoidosis in Japan. Eur Respir J 2008;31:372-379.

4 Cozier YC, Berman JS, Palmer JR, Boggs DA, Serlin DM, Rosenberg L: Sarcoidosis in black women in the United States: data from the black women's health study. Chest 2011;139: 144-150.

5 Rybicki BA, Major M, Popovich J Jr, Maliarik MJ, Iannuzzi MC: Racial differences in sarcoidosis incidence: a 5-year study in a health maintenance organization. Am J Epidemiol 1997; 145:234-241.

6 Judson MA, Boan AD, Lackland DT: The clinical course of sarcoidosis: presentation, diagnosis, and treatment in a large white and black cohort in the United States. Sarcoidosis Vasc Diffuse Lung Dis 2012;29: 119-127.

7 Baughman RP, Teirstein AS, Judson MA, Rossman MD, Yeager H Jr, Bresnitz EA, DePalo L, Hunninghake G, Iannuzzi MC, Johns CJ, McLennan G, Moller DR, Newman LS, Rabin DL, Rose C, Rybicki B, Weinberger SE, Terrin ML, Knatterud GL, Cherniak R; Case Control Etiologic Study of Sarcoidosis (ACCESS) Research Group: Clinical characteristics of patients in a case control study of sarcoidosis. Am J Respir Crit Care Med 2001;164:18851889.

8 Sharma SK, Mohan A, Guleria JS: Clinical characteristics, pulmonary function abnormalities and outcome of prednisolone treatment in 106 patients with sarcoidosis. J Assoc Physicians India 2001;49:697-704.
9 Ungprasert P, Carmona EM, Utz JP, Ryu JH, Crowson CS, Matteson EL; Epidemiology of sarcoidosis 1946-2013: a population-based study. Mayo Clin Proc 2016;191:183-188.

10 Rocca WA, Yawn BP, St Sauver JL, Grossardt BR, Melton LJ 3rd: History of the Rochester Epidemiology Project: Half a century of medical records linkage in a US population. Mayo Clin Proc 2012;87:1202-1013.

11 Zajicek JP, Scolding NJ, Foster O, Rovaris M, Evanson J, Moseley IF, Scadding JW, Thompson EJ, Chamoun V, Miller DH, McDonald WI, Mitchell D: Central nervous system sarcoidosis - diagnosis and management. QJM 1999;92:103-117.

12 Stern BJ, Krumholz A, Johns C, Scott P, Nissim J: Sarcoidosis and its neurological manifestations. Arch Neurol 1985:42:909-917.

13 Nozaki K, Judson MA: Neurosarcoidosis: clinical manifestations, diagnosis and treatment. Presse Med 2012;41:e331-e348. 
14 Stern BJ, Aksamit A, Clifford D, Scott TF: Neurologic presentations of sarcoidosis. Neurol Clin 2010;28:185-198.

15 Allen RK, Sellars RE, Sandstrom PA: A prospective study of 32 patients with neurosarcoidosis. Sarcoidosis Vasc Diffuse Lung Dis 2003;20:118-125.

16 Joseph FG, Scolding NJ: Neurosarcoidosis: a study of 30 new cases. J Neurol Neurosurg Psychiatry 2009;80:297-304.

17 Terushkin V, Stern BJ, Judson MA, Hagiwara M, Pramanik B, Sanchez M, Prystowsky S: Neurosarcoidosis: presentations and management. Neurologist 2010;16:2-15.

18 Sharma OP: Effectiveness of chloroquine and hydroxychloroquine in treating selected patients with sarcoidosis with neurological involvement. Arch Neurol 1998;55:1248-1254.
19 Cipriani P, Ruscitti P, Carubbi F, Liakouli V, Giacomelli R: Methotrexate: an old new drug in autoimmune disease. Expert Rev Clin Immunol 2014;10:1519-1530.

20 Ponticelli C, Moroni G: Hydroxychloroquine in systemic lupus erythematosus (SLE). Expert Opin Drug Saf 2017;6:411-419.

21 Baughman RP, Drent M, Kavuru M, Judson MA, Costabel U, du Bois R, Albera C, Brutsche M, Davis G, Donohue JF, Müller-Quernheim J, Schlenker-Herceg R, Flavin S, Lo KH, Oemar B, Barnathan ES: Infliximab therapy in patients with chronic sarcoidosis and pulmonary involvement. Am J Respir Crit Care Med 2006; 174:795-802.
22 Rossman MD, Newman LS, Baughman RP, Teirstein A, Weinberger SE, Miller W Jr, Sands BE: A double-blinded, randomized, placebo-controlled trial of infliximab in subjects with active pulmonary sarcoidosis. Sarcoidosis Vasc Diffuse Lung Dis 2006;23:201208

23 Toth C, Martin L, Morrish W, Coutts S, Parney I: Dramatic MRI improvement with refractory neurosarcoidosis treated with infliximab. Acta Neurol Scand 2007;116:259-262.

24 Cohen Aubart F, Bouvry D, Galanaud D, Dehais C, Mathey G, Psimaras D, Haroche J, Pottier C, Hie M, Mathian A, Devilliers $\mathrm{H}$, Nunes H, Valeyre D, Amoura Z: Long-term outcomes of refractory neurosarcoidosis treated with infliximab. J Neurol 2017;264: 891-897. 